\title{
Measuring Electrodermal Activity to Capture Engagement in an Afterschool Maker Program
}

\author{
Ryan Cain \\ Utah State University \\ 2830 Old Main Hill \\ +1 (718) 635-1625 \\ ryan.cain@aggiemail.usu.edu
}

\author{
Victor R. Lee \\ Utah State University \\ 2830 Old Main Hill \\ +1(435)797-7562 \\ victor.lee@usu.edu
}

\begin{abstract}
In this paper, we describe a new approach for exploring individual participants' engagement in youth maker activities. Participants were outfitted with wearable first person point-of-view still-image cameras and wrist-based electrodermal sensors. The researchers analyzed the recorded electrodermal data stream for surges in skin conductivity and compared them with the corresponding photographs based on their time-stamp. In following with prior work, these surges were interpreted as moments of engagement. A comparison sample was created to look at moments that lacked this psychophysiological marker. Results indicated that the two participants had both shared and divergent engagement with activities such as soldering, assembling, and programming.
\end{abstract}

\section{Categories and Subject Descriptors}

- Social and professional topics $\sim$ Computer science education • Social and professional topics $\sim$ Informal education

\section{General Terms}

Documentation, Human Factors

\section{Keywords}

Youth organizations, informal learning environments, makerspaces, afterschool clubs, electrodermal activity, wearables

\section{INTRODUCTION}

Making is thought to be highly engaging for a broad range of youth in well supported settings [1], [2]. An opportunity exists for researchers to better understand the ways in which individuals differentially engage with making. This is important to explore because making often provides an opportunity for customization of experience. What one youth finds engaging in a makerspace may differ from what another youth finds engaging. The current study aims to examine how youth makers respond psychophysiologically to some common maker activities, such as rapid prototyping and programming microcontrollers [3], in the context of a multi-week maker education program.

In addition to the methods we present in the following sections, all

Permission to make digital or hard copies of all or part of this work for personal or classroom use is granted without fee provided that copies are not made or distributed for profit or commercial advantage and that copies bear this notice and the full citation on the first page. Copyrights for components of this work owned by others than the author(s) must be honored. Abstracting with credit is permitted. To copy otherwise, or republish, to post on servers or to redistribute to lists, requires prior specific permission and/or a fee. Request permissions from Permissions@acm.org.

FabLearn '16, October 14 - 16, 2016, Stanford, CA, USA

Copyright is held by the owner/authors. Publication rights licensed to ACM.

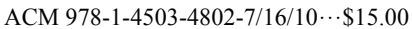

DOI: $10.1145 / 3003397.3003409$ the youth completed a survey at the end of each session to capture their overall level of engagement [4]. We observed a ceiling effect with the participants consistently reporting being highly engaged, $\mathrm{M}=27.44, \mathrm{SD}=3.18$ with $8-32$ as the possible range of scores. While this was encouraging to see such high engagement, the method below aims to elicit a more nuanced understanding of engagement.

In the following sections we present a new method we are exploring and initial findings from it using wearable cameras and sensors to examine engagement in maker activities at a minuteby-minute grain size.

\section{BACKGROUND}

\subsection{Engagement as a Phenomenon of Interest}

Engagement as a psychological and interactional phenomenon relies upon intuitive appeal. That is, we as a community of educational researchers make judgments based on observable behavior that suggests high levels of attention, intention, and participation. How it is operationalized and determined is still unspecified and subject to overarching theoretical perspectives that suggest how engagement is framed in relation to a given task or setting.

For the present work, we have drawn on a more established, but still developing body of work related to "interest". One of the most recognized theoretical models of interest comes from work by Hidi and Renninger [5]. Hidi and Renninger's four-phase model of interest development posits that interest progresses in the following stages: phase 1 - triggered situational interest; phase 2-maintained situational interest; phase 3- emerging individual interest; and phase 4-well-developed individual interest. We could imagine a youth enjoying a dinosaur exhibit among others on a walk through a museum-phase 1 . Upon the next visit to the museum the child asks to visit the dinosaur exhibit first-phase 2. While at the local public library, she checks out several dinosaur books and asks the librarian where to find additional resourcesphase 3. For career day at her school she presents a project about her future self as a paleontologist specializing in dinosaursphase 4.

Drawing on the four-phase model, moments of engagement would align with moments of situational engagement. That is, features of an activity that capture attention and elicit some seconds of consideration or participation from a youth are times we expect to see momentary engagement. It is entirely possible, and for us, expected that over many repeated encounters, those moments of engagement may become more enduring. Once an interest has sufficiently developed such that we ascribe a particular interest to a person, then we would expect to see that person continued and recurrent participation over longer periods of time with gaps in between. It is at that point we might say "Erica is really interested in dinosaurs". In extended and unbroken periods of engagement, 
attentional focus would be such that a person would experience the psychological phenomenon of "flow." In the example of Erica and dinosaurs, that might come about during several minutes of sketching a T-Rex or reading about the Apatosaurus genus.

However, it is the former that is our current focus. Situational engagement is something we posit to produce moments of heightened psychophysiological arousal. That arousal produces markers in the form of rapid and relatively immediate changes in skin conductivity. While there remains much still to be understood about skin conductivity changes in situ, we intend by way of this study to explore what can be revealed when rapid increases in skin conductivity, a signature of physiological arousal, are used as a proxy and marker for moments of situational interest and engagement.

\subsection{Electrodermal Activity}

The measurement of electrodermal activity (EDA), sometimes referred to as galvanic skin response, gauges psychophysiological activity of the sympathetic nervous system. Sensing EDA has long been a tool for measuring human psychophysiological responses to stimuli[6]. Arousal of the sympathetic nervous system produces a heightened sensitivity to environmental stimulation and results in increased intake and processing of information" p. 128 [7]. This response prepares the body for action [6] by signaling sweat glands on and near the hands and feet to produce sweat to increase friction for a better grip and traction respectively.

While electrodermal activation may not produce a visible amount of sweat at the surface of the skin, the electrical conductivity of the skin increases as the sweat glands begin producing sweat into the sweats duct as part of the autonomic response. The skin can be thought of as a sponge that becomes increasingly conductive as salty water fills its voids, which are the ducts that connect the sweat gland to the surface of the skin. This change in conductivity can be measured by passing a small current through the skin and measuring the resistance. The value is presented as the inverse of the resistance with the unit micro Siemens $(\boldsymbol{\mu S})$.

Pecchinenda [8] measured surges of EDA to indicate engagement, while participants were attempting to solve Tangram puzzles. The participants worked on solving puzzles of varied levels of difficulty finding participants stopped engaging as the puzzles presented became increasingly difficult with a corresponding decrease in EDA. Kreibig, Gendolla, and Scherer [9] similarly found a rise in EDA when their participants were given relevant feedback to help with the achievement of a goal. Both of these studies used EDA recording devices that required participants to be attached to a desktop computer via wires

The current study relies on wearable EDA recording devices to collect data unobtrusively allowing wearers to have full use of their hands without electrodes on their fingertips [10]. Additionally, participants wore wearable cameras to document what was in front of the person, providing context for the EDA data. This could include tools, computers, other participants, mentors, their hands assembling a circuit, etc. This affords a novel perspective into making by simultaneously monitoring external actions and internal psychophysiological activity.

\section{METHOD}

\subsection{Setting and Participants}

Two female participants ages 10 and 12 attended a twelve-week maker group at a youth makerspace in northern Utah. The participants $^{1}$ and an additional ten girls met once a week for two hours. Parent volunteers and makerspace employees mentored the girls with a child to adult ratio usually around 4:1. During these meetings they worked in pairs constructing a high altitude balloon, which they eventually launched and recovered.

The group followed a combination of activities developed by the group's leader, Christopher ${ }^{2}$, and the Ardusat online curriculum (ardusat.com/lessons, a space themed experiment platform for facilitating physical-computing based inquiry). These activities included building antennas for locating hidden radio transmitters, wiring sensors, and programming Arduino microcontrollers to read sensor values. Participant pairs were each responsible for an individual sensor that measured luminosity, ultraviolet light, infrared light, acceleration, orientation, or temperature. During the final weeks they installed the sensors, a radio beacon transmitter, a radio GPS transmitter, and an action camera into three 7" $\mathrm{x} 7$ " $\mathrm{x}$ 7 " foam boxes that they also built. The young women launched and recovered their sensor-laden boxes.. The balloon traveled to a height of over 110,000 feet and a distance that crossed a mountain range and state line.

\subsection{Arousal Terminology}

In the following sections we explain our procedures for preparing our data for analysis. A spike or sudden rise in skin conductivity indicates arousal of the sympathetic nervous system, as mentioned earlier. We use the terms arousing moments and unarousing moments to categorize times of rising and relatively flat levels of skin conductivity respectively. These two categories facilitate comparisons between the two in results section below. The time scale of a moment ranges from less than a minute to several minutes depending on the duration of the rising EDA.

\subsection{Data from Wearable Devices}

For this brief paper we present 3 days selected for their breadth of activities. We chose two out of the four girls who wore wearables since they worked as a pair and were in attendance at all sessions. Dot and Jane wore wrist based EDA sensors, Affectiva Q2, and point-of-view cameras, Autographer, around their necks. See Figure 2 for an image taken with the camera showing the Q2 EDA sensor on the girl's left wrist. EDA was recorded at $4 \mathrm{~Hz}$, see top of Figure 1. The cameras captured an image once ever 8-15 seconds based on its internal motion sensor. The wearable devices allowed the participants to freely move about while capturing EDA and still images.

\subsubsection{EDA Data Reduction}

Each of the two participants generated about 24,000 EDA datum per session $-4 \mathrm{~Hz}$ for 2 hours, see top Figure 1. We processed the data in $\mathrm{R}$ to develop a consistent way for identifying spikesindicating EDA arousal-while reducing noise. The arithmetic mean was calculated for the 40 datum per 10 -second intervalchosen to approximate the wearable cameras interval of a photo every 8-15 seconds-resulting in about 600 intervals. Each interval was subtracted from the following one in a $\Delta=X_{i+1}-X_{i}$ calculation resulting in what we will refer to as relative change in skin conductivity (RCSC). The 599 RCSC values are shown as dots on the bottom Figure 1 .

One positive SD of the distribution of RCSC values served as the threshold for defining arousing moments, indicating rising EDA

\footnotetext{
${ }^{1}$ Youth self selected into this optional after-school group.

2 All names have been replaced with pseudonyms to protect participants' identities
} 
to capture positive slopes that were not near zero. A randomly selected comparison set of unarousing moments was selected from RCSC values that were within $\pm .25 \mathrm{SD}$ because this selected values where the slope was near zero, see bottom of Figure 1. The resulting analysis contains two categories of moments, arousing and unarousing, shaded in light gray for arousing and dark gray for unarousing on the bottom of Figure 1.

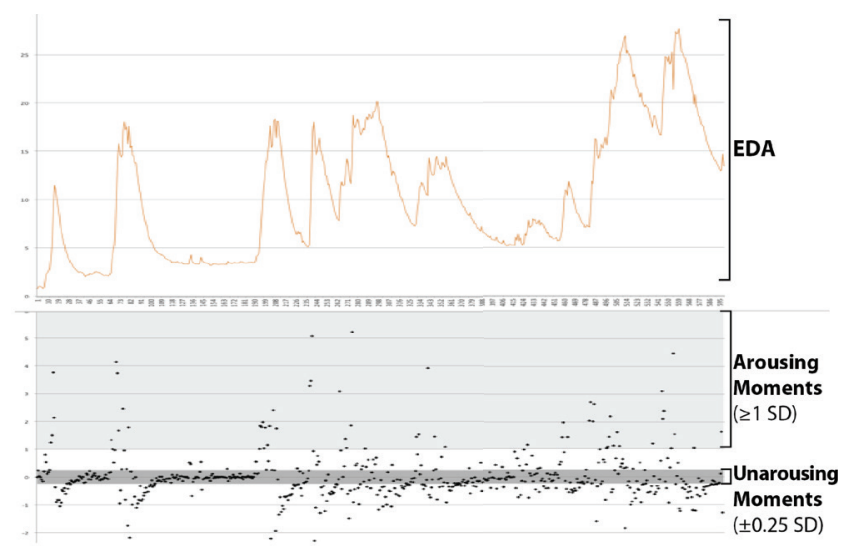

Figure 1 (Top) Electrodermal Activity for Dot on 9/8 (Bottom) deltas between 10-second intervals for Dot on 9/8. Light gray shading indicates arousing moments; dark gray shading indicates unarousing moments.

Table 1. Quantities of Arousing and Unarousing Moments with Quantities of Corresponding of Images in Parentheses

\begin{tabular}{lcclll} 
& \multicolumn{2}{c}{ Jane } & & \multicolumn{2}{c}{ Dot } \\
\cline { 2 - 3 } \cline { 5 - 6 } $9 / 8$ & Arousing & Unarousing & & Arousing & Unarousing \\
\cline { 2 - 3 } \cline { 5 - 6 } $9 / 8$ & $61(265)$ & $61(251)$ & & $51(245)$ & $61(217)$ \\
$9 / 15$ & $40(127)$ & $40(184)$ & & $37(150)$ & $40(179)$
\end{tabular}

Since our data reduction method defined arousing as greater than one SD and unaroused as within $0.25 \mathrm{SD}$ of the mean, we have excluded all other deltas that do not fit the criteria. This was a decision to conservatively define arousal as spikes excluding intervals when EDA is still high after a spike, but no longer rising. While this may ignore some engaging moments, we were most concerned when an activity elicited situational engagement, hence the greater attention to the surge. Additionally the intervals of descending EDA greater than $0.25 \mathrm{SD}$ are excluded as returning to baseline period. The quantity of these unarousing moments were set to be equal to the number of arousing moments for the participant with the greater number for that day. For example, on 8/11 Jane had 61 arousing moments and Dot had 51, the comparison unarousing moment set would be from 61 random moments $\pm .25 \mathrm{SD}$ for both participants. These unequal comparisons are accounted for in the image coding section below.

\subsubsection{Photo Inclusion Criteria}

The arousing and unarousing moments were used to select images from the wearable camera for illustrating the activity taking place. Both the EDA deltas and wearable images are time-stamped. Any image taken during a minute that contained an arousing moment was copied to a photo database for coding. The number of photos taken per minute varies due to the camera's decision-making algorithm based on motion and lighting conditions, but at a minimum of one photograph every 15 seconds.

\subsection{Data Coding}

\subsubsection{Coding Photos}

We developed a coding scheme to analyze the first-person perspective images from the wearable cameras. Initially the coding identified objects in the frame (Arduino, tool, laptop), setting (outside, workbench, walking), and people (mentor and participant names). We expanded the coding scheme to code for the actions taking place (assembling, testing, programming) when identifiable from the images. The action codes serve as a summary of the moment when identifiable. Typically the participant's hands or computer screen provide an identifiable action. For example, we observed Jane assembling in Figure 2.

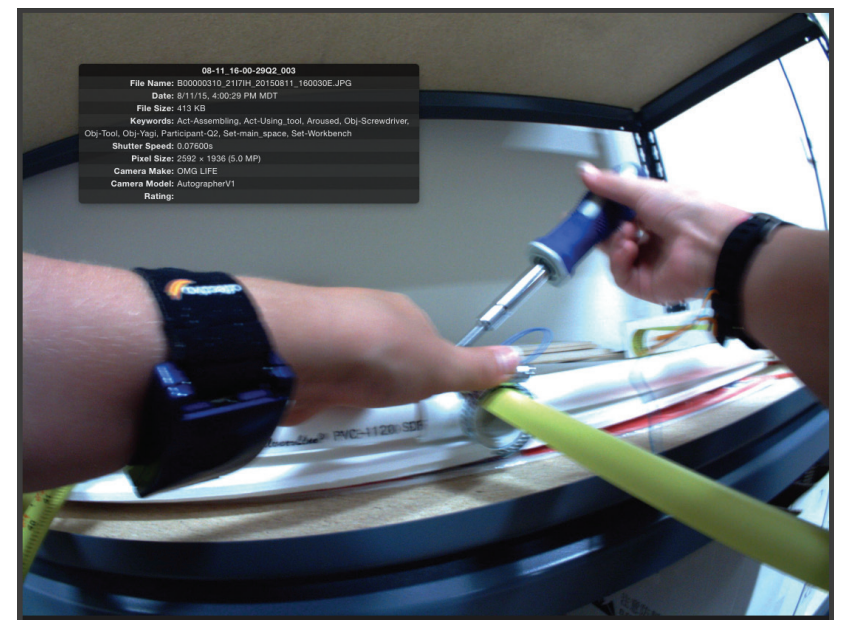

Figure 2. Coded image of Jane assembling an antenna with a screwdriver. The EDA wearable can be seen on her left wrist.

\subsubsection{Comparing Code Counts}

Once the images were coded for activities we wanted to make comparisons of counts between days and the two participants. In order to do this, we used proportional scaling to adjust for the unequal samples. The random sample of unarousing moments was based on the participant with the greater number of arousing moments. Additionally the wearable camera's algorithm for when to capture an image causes variability in the number of images taken per minute. Then we created the arousal ratio (AR) to define what activities were typically arousing. Events with a ratio $2: 3$ of unarousing moments to arousing moments or less ((unaroused event totals)/(aroused event totals) $\leq 0.66)$ were deemed typically arousing. For example we coded 47 aroused images and 16.94 unaroused images for Dot on 8/11 that yields an arousal ratio of 0.36 meeting our criteria for a typically arousing activity. We used these ratios to look across activities over the three days at where the two participants' arousal ratios (AR) coincided and diverged.

\section{RESULTS}

\subsection{Wearable Data Analysis}

The summary of arousal ratios, Table 3, revealed that Dot and Jane exhibited both shared and dissonant psychophysiological responses. There were times the two shared a common response like when both were aroused on 9/8 during images coded for the activity sharing out. The pair stood in front of their peers and mentors explaining their progress, so it's not surprising to see rises in EDA when it is common for presenters' hands to sweat. 
Here we are confident that they were engaged while sharing out. Other common responses for the pair included being typically unaroused when their arms were crossed on the table (code-hands resting) and while filling out the survey given at the end of every session.

Jane and Dot differ on 8/11 with arousal ratios (AR) of 0.75 and 0.36 respectively for the activity assembling. Both participants were working individually to build antennas on this day. As part of assembling the antennas, the participants soldered wires to connect the elements of the antenna together. We coded these images for both assembling and soldering. Dot was assembling a sensor on $9 / 15$ where she had 11 arousing moments ( $0.00 \mathrm{AR})$. While Dot was typically aroused by the act of assembling both on $8 / 11$ and $9 / 15$, she was less aroused by the activity of soldering. In contrast Jane was usually aroused while soldering (0.29 AR), but not quite during assembling (0.75 AR). Dot was split evenly between arousing and unarousing moments while coded for soldering (0.99 AR).

Table 3 Partial summary of arousal ratios for Jane and Dot over 3 days. Typically arousing activities are shaded in gray.

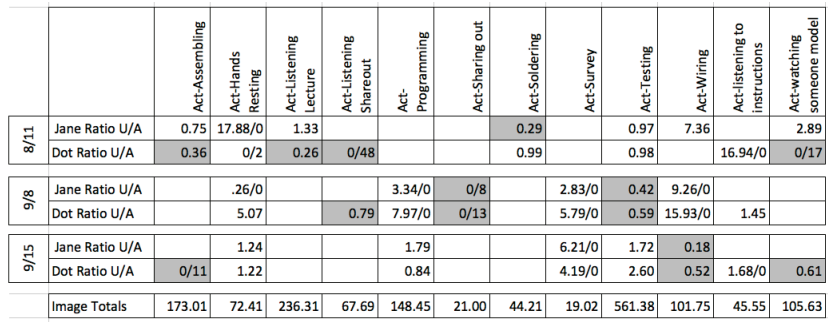

Looking at the activity of listening to lecture on 8/11 we see Dot was aroused with a $0.26 \mathrm{AR}$, while Jane was unaroused with 1.33 AR. On the same day, the code watching someone model shows a similar arrangement with Dot with 0.00 AR (0 unarousing moments/11 arousing moments). Additionally we see that Dot on a separate day, 9/15, has images coded for watching someone model, as in modeling how to solder, were typically arousing with a $0.61 \mathrm{AR}$. Our analysis of these four activity codes (assembling, soldering, listening to lecture, and watching someone model) begins to reveal some differences between how the pair of girls reacted differently to the shared activities.

\section{DISCUSSION}

In the previous sections we have argued that understanding engagement in a makerspace in a minute-to-minute scale could be valuable for looking at how learners engage with activities. While our method may detect times of heightened engagement, further work must test the validity of electrodermal arousal as a proxy for engagement. This is our initial analysis of the data set in order to develop the method. Further analysis is ongoing with the remaining nine sessions.

We captured unique arousal responses from Jane and Dot using the combination of a psychophysiological measure and firstperson point-of-view photos. While we did detect differences between the two, additional work is needed to examine engagement as a phenomenon of interest. This brief paper argues that our method is promising, but still a work in progress. We note our tentative assertion of identifying moments of arousal and engagement need significant justification as this work is more fully developed.

As making spreads into schools, libraries, and other community spaces, this method may assist researchers in understanding what practices individuals engage in.

\section{AKNOWLEDGEMENTS}

We thank Christopher, the mentors, and the participants for supporting this work. We are very grateful to: Joel Drake and Abigail Phillips for their feedback on drafts of this paper, Mac Cannady and Paulette Vincent-Ruz for their help with the survey, and Jeffrey Thayne for assisting with R. This work was supported in part by funding from the National Science Foundation under Grant No. CNS-1623401. The opinions expressed herein are those of the authors and do not necessarily reflect those of the National Science Foundation.

\section{REFERENCES}

[1] A. C. Barton, E. Tan, and D. Greenberg, "The makerspace movement: Sites of Possibilities for Equitable Opportunities to Engage Underrepresented Youth in STEM," Teach. Coll. Rec., 2016.

[2] V. Svihla, "Making for Engagement, Development and Learning," in the Preceedings of FabLearn 2015.

[3] L. Martin, "The Promise of the Maker Movement for Education," J. Pre-College Eng. Educ. Res. J. PreCollege Eng. Educ. Res. J. Pre-College Eng. Educ. Res., vol. 5, no. 5, pp. 1-30, 2015.

[4] A. Ben-Eliyahu, D. Moore, M. Bathgate, R. Correnti, S. Randol, R. Dorph, and E. Al, Investigating the Multidimensionality of Engagement: Affective, Behavioral, and Cognitive Engagement in Science Across Multiple Days, Activities, and Contexts," Contemp. Educ. Psychol.

[5] S. Hidi and K. A. Renninger, "The Four-Phase Model of Interest Development," Educ. Psychol., vol. 41, no. 2, pp. 111-127, 2006.

[6] R. R. Matsumoto, B. B. Walker, J. M. Walker, and H. C. Hughes, "Fundamentals of Neuroscience.," 1990.

[7] D. C. Raskin, Attention and Arousal. Academic Press, Inc., 1973.

[8] A. Pecchinenda, "The Affective Significance of Skin Conductance Activity During a Difficult Problemsolving Task," Cogn. Emot., vol. 10, no. 5, pp. 481-504, 1996

[9] S. D. Kreibig, G. H. E. Gendolla, and K. R. Scherer, "Goal Relevance and Goal Conduciveness Appraisals Lead to Differential Autonomic Reactivity in Emotional Responding to Performance Feedback," Biol. Psychol., vol. 91, no. 3, pp. 365-375, 2012.

[10] M. Z. Poh, N. C. Swenson, and R. W. Picard, "A Wearable Sensor for Unobtrusive, Long-Term Assessment of Electrodermal Activity," IEEE Trans. Biomed. Eng., vol. 57, no. 5, pp. 1243-1252, 2010. 